\title{
ACCIONES DE MAESTROS DE CIENCIAS: CREENCIAS, ROLES, METAS Y CONTEXTOS EN LA ENSEÑANZA Y EL APRENDIZAJE
}

\author{
Lilia Reyes Herrera Ph.D.* y Luis Enrique Salcedo Ph.D.*
}

\begin{abstract}
Although scence curricula vary widely among countries, states, school districts, individual schools and individual classrooms, the understanding and enhancement of science teaching and learning is so limited in most of them that it is a global concern. Teacher's conceptions play an important role in the implementation of a science curriculum, consequently; the study and development of teacher conceptions of the nature of science education has become one of the most important goals of the science education community. Although in the last twa decades this has been a prolific area of research, it is yet to be researched more deeply. The overallpurpose of this research is to explore the nature of the referents used by science teachers when engaged in science instruction and to elucidate patterns of beliets, goals, roles and context which guide teachers actions and interactions. The kind of research we are doing argue tora change in teachers' epistemology empowering them to take informed decisians to break away fram dominant practices which have been present in the community without deep questioning.
\end{abstract}

El diseño y desarrollo de esta línea de investigación responde a la necesidad de consolidar procesos diferentes para la enseñanza y el aprendizaje de las ciencias en nuestro país En particular pretende ser una estrategia de formación capacitación y fortalecimiento de recursos humanos de alta calidad investigativa para el desarrollo de la investigación en este campo. La estructura de esta línea integra coherentemente investigaciones en el campo de la educación en ciencias que proveen elementos para ampliar las fronteras del conocimiento y dar posibles respuestas a lo que está sucediendo actualmente en la mayoría de las clases de ciencias.

Se propone además como un espacio de exploración, reflexión, análisis y construcción de conocimientos en torno a las relaciones entre las acciones de los profesores de ciencias y sus referentes filosóficos, epistemológicos, sociológicos y disciplinares En la medida en que por medio de la investigación educativa, se construyan nuevos significados acerca de las creencias, roles, metas y contextos en los cuales se halla embebida la acción del docente, ya sea en la educación básica, media o superior, se abrirán nuevas perspectivas de interpretación y acción de las prácticas pedagógicas en ciencias. Mediante el desarrollo de esta investigación se pone en evidencia de manera documentada la necesidad de introducir cambios en los procesos de enseñanza y de aprendizaje que respondan de manera más coherente a los problemas que presenta la educación en ciencias en nuestro país.

La investigación pedagógica en el área de las ciencias realizada en los últimos años ha permitido conocer los principales problemas que se presentan en los procesos de

\footnotetext{
* Profesora asociada Universidad Pedagógica Nacional Departamento de Biología.

** Profesor asociado Universidad Pedagógica Nacional. Departamento de Química.
} 
enseñanza y aprendizaje especialmente en el nivel secundario. Se ha señalado que la introducción de conceptos se realiza de manera arbitraria, sin tener en cuenta las ideas previas de los alumnos (Carrascosa, 1987; Salcedo 1991) y además haciendo énfasis en definiciones y en aplicaciones de fórmulas matemáticas. Por otra parte, las prácticas de laboratorio no contribuyen a familiarizar a los estudiantes con las características del trabajo científico ni al fortalecimiento de aprendizaje significativo de conceptos (Payá, 1991 Salcedo, 1994). La resolución de problemas de lápiz y papel tan ampliamente utilizada parlas profesores de Química, como recurso didáctico no favorece cambios metodológicos y conceptuales en los estudiantes (Gil, 1988; Calderón, Prieto y Ruiz, 1994). En este contexto, tópicos tales como la evaluación de la enseñanza y del aprendizaje han recibido poca atención por parte de los investigadores y se plantea la urgente necesidad de realizar estudios en este campo (Salcedo y otros, 1996).

A nivel universitario la investigación pedagógica en ciencias ha sido muy esporádica y parece reproducirse la situación encontrada en el nivel secundario. En particular, nos interesa abordar la situación de las instituciones formadoras de docentes, pues sin duda las fallas que allí se presentan inciden directamente en la calidad de la educación en ciencias a nivel secundario. En este contexto el desarrollo del campo de investigación relacionado con el profesorado ha sido de particular importancia.

Partimos del supuesto de que los profesores de ciencias tienen sus propias concepciones (formas de pensamiento, epistemologías, creencias) sobre el conocimiento, la ciencia (Pope \& Scott, 1983), la enseñanza (Hewson \& Hewson 1987), el aprendizaje (Aguirre, Haggerty \& Linder, 1990) y demás aspectos inherentes a la educación en ciencias, que influyen de una u otra manera en su actuación en el aula y que incluso pueden entrar en conflicto con los resultados mayoritariamente aceptados en la investigación educativa.

Se trata entonces de estudiar el pensamiento de los profesores de ciencias específicamente sus epistemologías con el fin de posibilitar los necesarios cambios que efectivamente contribuyan al mejoramiento de la Educación en ciencias en nuestro medio. Este planteamiento se hace ya que muchos profesores de ciencias le asignan mayor importancia a la transmisión y asimilación de conocimientos que a la compresión de la naturaleza de las ciencias (Hodson,1985), y al desarrollo de actitudes científicas y actitudes hacia las ciencias (Schibecci, 1982).

Hay evidencia de que muchos profesores de ciencia poseen y transmiten una visión de carácter empirista-inductivista de las ciencias con frecuencia asociada a una visión de carácter mítico-mágico. En este contexto, la enseñanza se considera como actividad que clarifica los contenidos que se presentan en los textos, que explica la solución de problemas" (ejercicios de aplicación a final de capitulo) como comprobación de leyes y teorías a través de las prácticas de laboratorio Estos profesores frecuentemente conciben el proceso de aprendizaje como de asimilación solitaria y memorística (la mente como tabula rasa), pasiva y acritica de los nuevos conocimientos a la estructura cognoscitiva que ya se posee (Porlán 1989) y el proceso de enseñanza como una actividad centrada en la transmisión de contenidos.

Ante esta situación, se evidencia una urgente necesidad de replantear las bases epistemológicas, sicologógicas y didácticas de los currículos de ciencias, a la luz de planteamientos actuales de la filosofía de las ciencias, concretamente de la epistemología, de la sicología cognitiva y de los resultados de las investigaciones en estos 
campos, así como también se hace necesario un enfoque diferente en los programas de formación inicial y permanente de profesores de ciencias.

La formación inicial y permanente del profesorado ha de plantearse en íntima relación con los avances de la investigación pedagógica y con una aspiración teórica, es decir con el objetivo explícito de mostrar el carácter de cuerpo coherente de conocimientos alcanzados por la investigación en la educación de ciencias (Furia, 1992); pues existe un grave peligro de que las profundas transformaciones asociadas a las orientaciones constructivistas hoy emergentes queden desvirtuadas en su aplicación en la práctica (Gil, 1991). No basta con diseñar cuidadosa y fundamentadamente un currículo, si el profesorado no ha recibido la formación adecuada para desarrollar su labor educativa orientada por los principios epistemológicos, sicológicos y didácticos del currículo propuesto y si sus concepciones sobre las ciencias, su enseñanza y su aprendizaje están en conflicto con tales principios (Briscar, 1991) De esta manera los programas de formación inicial y permanente de profesores de ciencias deben propiciar el estudio y la evolución de las concepciones sobre las ciencias, su enseñanza y su aprendizaje para actuar consecuentemente de tal manera que el profesor pueda aproximar su trabajo docente al logro del progreso intelectual, moral, ético y social de sus alumnos generando en ellas evoluciones conceptuales, metodológicas y actitudinales.

Los profesores, principalmente con experiencia, tienen normas y principios de actuación en la práctica que guían su planificación y su conducta interactiva. Estas normas y principios configuran un sistema dentro del pensamiento del profesar a la manera de creencias. Por lo tanta, si consideramos que el papel del profesar debe ser un mediador entre la cultura y las estudiantes utilizando el curriculum, entonces adquieren un papel de primera importancia las concepciones de las profesares en la modelación de los contenidos $\mathrm{y}$, en general, todas aquellas perspectivas profesionales que se relacionan más directamente con las decisiones que el profesar toma cuando lleva a cabo su .práctica pedagógica. Las creencias del maestra serán en parte, responsables de los significadas que él atribuye a las componentes del curriculum y a las formas de desarrollarla, sea cual sea el grado de definición con que este se presente.

\section{Propósito de esta línea de investigación}

El propósito de este estudio es explorar las acciones de los profesares y de las estudiantes en aulas de clase de ciencias de diferentes gradas del sistema escalar y analizar cómo estas acciones están influenciadas por referentes derivados de su historia personal y del grupo social al cual pertenecen. Se dará especial consideración:

1 A la filosofía personal que posee cada maestro en torno al por qué, al qué y al para qué del conocimiento científico, de la enseñanza y del aprendizaje.

2. A referentes sociales asociadas con las formas en las cuales el maestra construye los ambientes para el trabaja de sus estudiantes.

3. A los conocimientos personales sobre las disciplinas especificas y en particular a sus ideas y acciones sobre prácticas de laboratorio, resolución de problemas, actitudes hacia la ciencia y el aprendizaje, evaluación de la enseñanza y aprendizaje de las ciencias y relaciones entre ciencia, tecnología y sociedad. 
El entender por ejemplo las creencias de profesores acerca de la naturaleza de la ciencia abrirá caminos que facilitarán el entendimiento de la práctica de maestras en diferentes contextos colombianos, pues en la medida en que el maestra cuestione qué entiende por ciencia; reflexione y se apropie de sus concepciones, podrá trabajar científicamente y asumir roles coherentes, cama también crear expectativas e incentivar actitudes apropiadas en sus estudiantes hacia el aprendizaje de la ciencia (Reyes, 1993).

\section{Importancia de esta línea de investigación}

La investigación en esta línea es importante al menos par das razones: Primera, parece ser una de los primeras estudios sistemáticos de investigación interpretativa realizado en aulas de enseñanza de ciencias en Colombia que, desde el punta de vista de los maestras investigadores comprometidas, analiza holísticamente sus propias acciones e interacciones. Segunda, se sugieren tres importantes hipótesis cuya comprobación basada en evidencia proveniente de los datos de la investigación apartará elementos indispensables para el mejoramiento de la enseñanza de las ciencias.

Estas hipótesis son.

- Los desequilibrios de poder entre el maestro y el estudiante representan importantes obstáculos para el aprendizaje de los estudiantes.

- Planteamientos filosóficos coherentes con el empirismo dificultan la enseñanza y el aprendizaje de las ciencias. Estos planteamientos condicionan las acciones de los profesores de ciencias.

- Al experimentar estrategias de formación inicial y permanente del profesorado, será posible transformar las acciones de los profesores de ciencias

Parlo tanto la reflexión crítica de la propia acción de los maestros constituye una valiosa herramienta intelectual para el avance de la reforma de la educación en ciencias.

\section{El Problema}

El problema central de este estudio es analizar referentes (culturales, sociales. epistemológicas, filosóficos, metodológicos) de acciones y cómo estas acciones e interacciones del maestro limitan a favorecen el aprendizaje de los estudiantes y de los mismas maestros. Este estudio también analiza la influencia de la retención por parte de las maestras de formas establecidas de enseñanza que pueden inhibir su propia habilidad para introducir procesas de cambio. Par lo tanto, como resultado de estas acciones los estudiantes no están adecuadas y oportunamente preparadas para tener éxito en esta sociedad que evoluciona tan rápidamente en materia científica y tecnológica.

La construcción de una visión teórica por parte de cada profesar puede proveer un punta de partida para iniciar las cambios requeridas en la práctica educativa y en la reforma de la enseñanza de las ciencias hacia el mejoramiento de las ambientes de aprendizaje de las estudiantes. La visión teórica del maestra trabajará coma un marco de referencia y cama una guía que oriente las acciones del maestra de ciencias. 


\section{PREGUNTAS DE INVESTIGACIÓN}

Las respuestas a las siguientes preguntas son importantes consideraciones que contribuyen a elaborar una marca de referencia para pensar y actuar acerca de la educación en ciencias: ¿Cuáles son los referentes básicos de las acciones del maestro? ¿Cómo estos referentes se ven afectados por los contextos en las cuales se presentan las acciones del maestro? ¿Cómo se ven afectadas las acciones del maestra parlas creencias que consciente a inconscientemente posee? ¿Cuál es la relación entre las acciones del maestro y las metas que él a ella han identificada para el aprendizaje de sus estudiantes? ¿En qué medida las propósitos del maestra inherentes al desarrolla de su práctica son coherentes can sus creencias en los contextos en los cuales el curriculum de ciencias es desarrollado? ¿Cuáles son las interacciones entre el sistema social del aula de clase y otras sistemas de influencia que también limitan las acciones del maestro? Para responder estas y otras preguntas relacionadas, nos proponemos el desarrolla de proyectos tales como:

1. Relaciones entre creencias del maestro y construcción de conocimiento en salones de ciencias en secundaria.

2 Concepciones de ciencia, enseñanza y aprendizaje en programas de química, física y biología.

3. Influencia de referentes constructivistas en el cambio de roles en maestros y estudiantes de ciencias a nivel secundario

4 Contextos de aprendizaje en un salón de ciencias del quinto grado de primaria

5 Impacto de mitos culturales en el aprendizaje y enseñanza de las ciencias.

6 Principios epistemológicos del pensamiento disciplinar y pedagógico de maestros

\section{CONTEXTO DE ESTE ESTUDIO}

A comienzos de la década del noventa profundos cambios socio-políticos en Colombia involucraron nuevas direcciones, nuevos retos y nuevos compromisos para el proceso educativo. Como resultado de estos nuevos planteamientos socio-políticos y del análisis de necesidades del pueblo colombiano se promulgó una nueva Constitución Política Nacional (Colombia, Congreso de la República, 1991) Esta situación dio origen también a una nueva Ley General de Educación que guía y organiza la educación en el país (Colombia, Congreso de la República, 1993)

Contexto especifico. Colombia tiene una población de cerca de 37 millones de habitantes y un área geográfica de 1.139 .000 kilómetros cuadrados. Es considerado como un país de ingreso medio can un promedio de inflación del $25 \%$. De acuerdo con Rodríguez (1988), $86 \%$ de la educación primaria, $62 \%$ de la secundaria y $3 \%$ de la educación preescolar es suministrada por el gobierno El ministro de educación es designado por el presidente de la República y la mayoría de las políticas educativas del país provienen del Ministerio de Educación Nacional Los estudiantes se mueven a través de niveles en su educación formal un año de preescolar, nueve años de educación básica (cinco primaria y cuatro secundaria), dos años de educación media vocacional y cuatro o 
cinco años de educación superior en una universidad La educación no-formal no sigue un curriculum secuencial y no conduce a títulos formales.

De acuerdo con Erazo (1996), el modelo educativo que ha predominado en nuestro país se caracteriza por "una visión simplista y reduccionista del proceso educativo, dentro del cual el conocimiento es producido y desarrollado par otros, en otras latitudes y para adquirirla sólo se requiere procesos de transmisión-asimilación, catalizados por profesores, en las instituciones educativas creadas casi exclusivamente para tal efecto" (p. 3). La capacidad de producción y desarrollo propio del conocimiento ha sido subvalorada en nuestra medio con gran detrimento para la capacidad de autogestión. Esta situación contribuye a prolongar nuestra dependencia de concepciones foráneas llevándonos cada vez más a perpetuar el modelo de sociedad de consumo que depende del desarrollo de bienes y servicios educativos de otros países y nos lleva a conformarnos con la triste alternativa de adaptar modelas extranjeras que en la mayoría de los casas no satisfacen nuestras necesidades ni se ajustan a nuestros patrones culturales. Una verdadera educación de calidad en nuestra medio implica desarrollo de modelas propias que respondan a la idiosincrasia de nuestras comunidades y que favorezcan la autonomía y el auto desarrollo de los estudiantes y demás agentes educativos.

Por ello uno de los retos que afronta la educación actual en Colombia, es la formación de recursos humanos con capacidad de producción de conocimientos científicos y tecnológicos para enfrentar sus problemas y producir nuevos bienes y servicios, a fin de asegurar el bienestar de su población y participar en la dinámica mundial como nación independiente y competitiva Por tanto requiere la apropiación social de la ciencia y la tecnología y la formación de ciudadanos capaces de comprenderlas y producir conocimientos en estas áreas (CONPES, 1994).

El problema de la educación no radica propiamente en que las escuelas estén trabajando peor que en el pasada. Lo que puede suceder es que las demandas del mundo y de la saciedad actual son tan grandes que la escuela no puede dar respuestas satisfactorias a esas demandas y preparar adecuadamente a los estudiantes para vivir una vida productiva en un mundo que cambia velozmente debida a los grandes avances tecnológicos. También puede suceder que a pesar de que la mayoría de maestras hablan sobre el cambio y reconocen la necesidad de cambiar, ellas no pueden cambiar o el cambio que se da no es efectiva ya que la mayoría de ellas no posee referentes ni imágenes necesarias para implementar y sostener las cambios anhelados (Reyes, 1993). Sin embargo los investigadorés educativos han demostrado que hay alternativas a las formas tradicionales de pensar y actuar en la enseñanza y aprendizaje de las ciencias. Una de estas alternativas es la de acoger los planteamientos canstructivistas y emprender investigaciones que proporcionen al maestra la fortaleza necesaria para reflexionar en su propio aprendizaje y en la construcción de mejores ambientes de aprendizaje para sus estudiantes.

\section{MARCOS CONCEPTUALES}

En la construcción del marca teórico para esta línea de investigación hemos tomado proposiciones de cuatro tendencias teóricas: el constructivismo con su enfoque radical y socio-cultural; los enfoques para el análisis de los sistemas sociales desde el punto de vista de los actores y de la saciedad; la teoría de la acción holística y las orientaciones de la pedagogía crítica. 


\section{Constructivismo}

En este estudio acogemos la posición filosófica del constructivismo con el entendimiento y la convicción de que el constructivismo no debe ser considerado como una nueva verdad para reemplazar planteamientos objetivos. Por el contrario, el constructivismo es una forma de pensar acerca del conocimiento, un referente para construir modelos de aprendizaje, de enseñanza, de renovación curricular (Tabin, 1993). Glasersfeld (1995; p. 51) sugiere las siguientes principias cama fundamentos de la epistemología constructivista radical:

- El conocimiento está en la mente de las sujetos que conocen y la que el ser humana conoce ha sida construido a partir de la experiencia previa.

- El conocimiento no es recibido pasivamente a través de los sentidos o por medio de la transmisión. Por el contrario el conocimiento es activamente construido por el sujeto que conoce.

Estos planteamientos son de gran importancia para el desarrollo curricular; por ejemplo, la idea de curriculum de interés emancipatorio explorada por Grundy (1987) proporciona una perspectiva promisoria para analizar aspectos fundamentales del constructivismo crítico tales como la construcción del poder, la emancipación, la liberación, la concientización. La idea central es liberar a los maestros y a los estudiantes de las ataduras de ideas dominantes que han enraizado normas sociales y reglamentos en los salones de clase de ciencias por muchos años. La mayoría de estas normas han sido aceptadas por mucho tiempo y pasadas de generación en generación sin haber sido nunca cuestionadas en cuanto a su relevancia y su pertinencia. Debido a que la enseñanza y el aprendizaje son acciones culturales embebidas en culturas particulares derivadas de una cultura central; el entendimiento de la cultura es una prioridad para darle sentido a las creencias, propósitos y haceres del maestro (Reyes, 1996).

\section{Análisis de Sistemas Sociales}

Galtung (1980), considera das perspectivas para examinar sistemas sociales: la perspectiva orientada a la consideración de los actores individuales y la perspectiva orientada a la consideración de la estructura de la sociedad. Existen das principales fuentes de poder interactuando en el aula de clase que contribuyen a un entendimiento integral de la enseñanza y el aprendizaje de las ciencias. La una resulta de la consideración de los actores particulares, las acciones e interacciones entre ¡os participantes y las intentos de negociación de significadas. La segunda fuente de poder se origina a partir de la consideración de la estructura total del macro-sistema social en el cual están embebidos las participantes.

Galtung, sugiere el análisis de indicadores sociales como referentes de la coherencia y permanencia de la sociedad deseada. Por lo tanta una revisión del enfoque de Galtung es relevante en el análisis de las acciones e interacciones en el aula de clase y la escuela, su enfoque es asumida en esta investigación.

\section{Una teoría de acción holística}

Uno de los puntos clave de esta investigación es el hecho de que es presentada desde la perspectiva de los actores. Por lo tanta lo aprendido de esta investigación está 
relacionado y refleja directamente la forma en que el maestro y el investigador piensan y actúan. Por consiguiente las acciones del maestro son estudiadas desde su propia perspectiva como autor. Generalmente muchos maestros perciben "acción" como sinónimo de comportamiento. Sin embargo en este estudio el significado de acción es diferente, el término "acción" adquiere un mayor significada del cual el comportamiento es solamente una parte. El comportamiento puede ser considerado en esta investigación como una conducta sin propósito específico o como una forma de hacer", mientras que la "acción" en esta investigación es entendida como un concepto holístico que puede ser pensada como un grupo de interacciones dialécticas que involucran los propósitos de un individuo, las creencias de que ciertas comportamientos son viables en un contexto determinado, la construcción individual del contexto en el cual la acción está embebida y el comportamiento del individuo" (McRobbie \& Tobin, 1995, p. 381). Por lo tanto, creencias, propósitos, comportamientos y contextos cuando están coordinadas de manera significativa y coherente permiten al maestro empezar a darse cuenta de la forma en la cual él o ella desarrolla sus acciones.

\section{Pedagogía crítica}

Contribuye a la discusión de aspectos de gran relevancia en el análisis de tensiones sociales. Analiza desequilibrios en asuntos tales como relaciones de discurso y poder que están afectando la forma en la cual el maestra realiza un curriculum específico. Este estudio también introduce una nueva visión de curriculum y de escuela como comunidades de diálogo donde la autonomía, el poder y el control son negociados a través del diálogo, y el discurso de cada uno de los participantes es escuchado y valorado. Aspectos relacionados con autonomía, poder y control son también considerados como fuerzas sociales que limitan la acción de los maestros.

Freire (1993) nos da elementos para desarrollar un nuevo pensamiento acerca de la educación emancipatoria y del proceso liberador de la educación. La investigación del pensamiento de los maestros para encontrar la razón de ser de su práctica educativa a través de la reflexión crítica debe tener sus raíces en a comunidad. Tenemos la expectativa de que con esta exploración de sus razones cada uno de los maestros se enriquecerá de un profundo y significativa entendimiento a cerca de cómo construir-se a sí mismo como maestro. Su propia construcción, como investigador de su propia acción educativa se ve también enriquecida en ja medida en que el maestro sea capaz de construir teoría acerca de su propia proceso educativo (Reyes-Herrera, 1996). Una de las argumentaciones teóricas que favorecen esta visión es el cambio de paradigmas que involucra una visión más crítica, una reflexión y un mayor compromiso del maestro consigo misma con sus estudiantes y con su escuela. Este compromiso exige el desarrollo de una práctica educativa más humana y de mejor calidad en la escuela

\section{METODOLOGÍA}

\section{Naturaleza de datos}

Esta línea de investigación etnográfica será desarrollada siguiendo los criterios de calidad propuestos por Guba and Lincoln (1989). Los datas de esta investigación provendrán de una permanencia intensiva en aulas de clase de los maestras estudiados mientras desarrollan sus clases de ciencias con sus estudiantes en grados de educación básica media o superior. Fuentes importantes de datas serán natas diarias de campo, 
video-grabaciones entrevistas formales reportadas en audio grabadora, memorandos y otros documentos pertinentes. También se buscará la oportunidad de hablar informalmente con los maestros cada momento en que la situación sea propicia y necesaria. Los maestros tendrán la oportunidad de ofrecer sugerencias y comentarios acerca de lo que se esta aprendiendo de la investigación. De esta manera los aprendizajes posteriores se beneficiarán de lo aprendido anteriormente.

\section{Criterios para juzgar el mérito de esta investigación}

\section{A. Criterio de verdad}

Este criterio es paralelo al criterio positivista de rigor.

Contempla:

1 Credibilidad

2. Transferencia.

3 Dependencia.

4 Confirmabilidad

Desde un punto de vista constructivista, credibilidad es un criterio más apropiado que el de validez interna, hace referencia a la coherencia entre la realidad de los actores y las construcciones atribuidas a ellos Algunas de las técnicas que incrementan la credibilidad son: compromiso y permanencia prolongada, observación persistente, discusión de pares, revisión y discusión de los mismos actores (Guba \& Lincoln. 1989, p. 237) Transferencia es el criterio cualitativa que equipara la validez externa o generalización de los datos. Una abundante" descripción o descripción minuciosa y detallada es la principal técnica para establecer condiciones significativas que facilitarían a otras personas que así lo deseen, juzgar la meritoriedad y aplicar lo aprendido en este estudio a su propia situación y contexto" (p. 242).

La dependencia está relacionada con la estabilidad de los datos en un periodo de tiempo determinado. Los datos dependen del diseño metodológico.

La confirmabilidad del proceso de investigación asegura que los datos, interpretaciones y resultados del proceso están centrados en contextos y personas diferentes del evaluador.

\section{B. Proceso Hermenéutico}

Un segunda criterio para juzgar el mérito de una investigación constructivista es el proceso hermenéutico. Este proceso consiste básicamente en un meta-análisis que implica una auto-evaluación. Uno de los beneficios de este proceso hermenéutico está representado por la posibilidad de una retroalimentación continua e inmediata de las interpretaciones. Por lo tanto errores que no han sido detectados antes, no tienen mayor chance de permanecer ocultas por largo tiempo.

\section{Autenticidad}

Un tercer gran criterio para juzgar la meritoriedad de una investigación cualitativa se relaciona con la autenticidad. Hace referencia al grado en el cual lo que está sucediendo en el aula de clase de enseñanza de las ciencias es representado textualmente par el 
investigador. Este criterio de autenticidad requiere que lo descrito corresponda con lo sucedido, también hace referencia al grado de coherencia entre las construcciones autónomas del investigador y su estructura de valores. La autenticidad involucra evolución, mejoramiento, madurez, elaboración de información y uso de nuevas conocimientos por parte de los participantes tal como lo describen Guba y Lincoln (1989).

\section{BENEFICIOS DE ESTA LÍNEA DE INVESTIGACION}

Ya que el propósito fundamental de esta investigación es explorar los referentes que guían las acciones e interacciones de maestros de ciencias, se da especial atención al análisis de referentes sociales y mitos culturales que puedan incidir en la construcción de ambientes de aprendizaje en dominios tales coma la Biología, la Química, la Física y otras ciencias de la vida y ciencias de la tierra. Cuidadosas investigaciones interpretativas pueden ofrecer a los maestros, administradores y directivas que las desarrollen grandes posibilidades para reconocer la existencia de poderosas fuerzas sociales en sus comunidades, que actúan como mitos culturales limitando las acciones e interacciones del maestro. La reflexión y pensamiento critico puede asistir a los maestros en la identificación y demitificación de mitos culturales y en la iniciación de esfuerzos de reforma educativa tendientes a beneficiar el aprendizaje de las ciencias Si los maestros pueden identificar los mitos que sirven de referentes a sus prácticas educativas un análisis profundo de estos referentes podría llevar a los maestros a generar formas alternativas de pensamiento acerca de la enseñanza y el aprendizaje y a proponer y comprometerse con cambios correspondientes que favorecerán reformas curriculares.

\section{Implicación de esta investigación}

Un aspecto importante que merece especial relevancia es el modelo de investigación cualitativa que ofrece este estudio a las maestros de ciencias que desea' realizar investigación interpretativa, etnográfica acerca de sus propias propósitos y creencias concernientes a la ciencia, el aprendizaje, la enseñanza y la investigación. Este enfoque investigativa enriquece el entendimiento de lo que está sucediendo en el aula de clase teniendo en cuenta los factores socio-culturales saciados con la enseñanza de la ciencia en las comunidades.

De acuerdo con Reyes (1996), poderosas fuerzas sociales dominantes expresadas en términos de desequilibrios de poder y enfoque de enseñanza-aprendizaje que consideran la realidad independiente de la mente en términos de verdades que deben ser aprendidas de manera inflexible y general, limitan grandemente la forma en la cual las ciencias son aprendidas en numerosas aulas de clase. Esta línea de investigación reflejará claramente cómo el mito de la visión objetiva permea intensivamente los esfuerzos de cambio por parte de muchos maestras de ciencias de la vida y ciencias de la tierra entre otras disciplinas La interpretación de los marcos teóricos que emergen de esta investigación pueden apoyar los propósitos de cambio de los maestros y el proceso de reforma curricular en el área de ciencias. Muchos maestros encontrarán importantes argumentos para reflexionar y decidir acerca de sus propias acciones, y los directivos podrán encontrar elementos significativos que apoyen sus propósitos de satisfacer las necesidades de los maestros y el cambia curricular.

En el contexto de Colombia, país que lucha por sobrevivir muchas crisis, compartimos la expectativa de que lo que se aprenda de esta investigación proporcionará visiones útiles a los educadores de ciencias que les ayudará a reflexionar en su propia práctica 
abriendo futuras direcciones para la enseñanza de las ciencias no solamente en la escuela sino en todas aquellas instituciones sociales en las cuales la escuela y el salón de ciencias están inmersas. Las implicaciones de este estudio presentan un gran potencial para el mejoramiento de áreas educativas en ciencias tales como: aprendizaje, enseñanza, cualificación del maestro, formación de maestros, política educativa, reforma educacional, desarrolla curricular y desarrollo de la investigación educativa.

\section{REFERENCIAS}

AGUIRRE, J., Haggerty, S., \& Linder. C. (1990). Student-teachers conceptions of science, teaching and learning: a case study in preservice. Science Education, pp. 380-390.

BRISCOE, C. (1991). The dynamic interactions among beliefs, role methaphores and teaching practices. A case study of teacher change. Science Education, 75(2), pp.185199.

CAICEDO, H. (1992). Viabilidad de una línea de investigación sobre enseñanza de las ciencias. Revista Colombiana de Educación, 24, 91-98.

CALDERÓN, J.; Prieto, C. y Ruiz, D. (1994). Resolución de problemas como investigación para la familiarización con la metodología científica y el aprendizaje significativo del concepto de reacción química. Tesis de grado. Universidad Pedagógica Nacional, Bogotá, Colombia.

CARRASCOSA, J. (1987). Tratamiento didáctico en la enseñanza de las ciencias de los errores conceptuales. Tesis doctoral. Universidad de Valencia, España.

COLOMBIA, Congreso de la República (1991). Constitución Política Nacional de Colombia).

COLOMBIA, Congreso de la República (1993). Ley 60 de 1993. Ministerio de Justicia. Diaria Oficial. Imprenta Nacional. Santafé de Bogotá, D.C., Colombia

CONPES. (1994). Política Nacional de Ciencia y Tecnología. Departamento Nacional de Planeación. El Salto Social. Documento 2739. Santafé de Bogotá.

ERAZO, M. (1996). El capital humano y la calidad de la educación. Editorial. En Apuntes Pedagógicos. Universidad Pedagógica Nacional. Santafé de Bogotá, D.C., Colombia.

FREIRE. P. (1993) Pedagogy of the oppressed. (M, Bergman Ramos, Trans.) New Revised 20 th-Anniversary. Ed. New York: The Continuum. Publishing Company. (Original Work published 1970).

FURIO, O., Gil, D., Pessoa, A., y Salcedo L. E. (1992). "La formación inicial del profesorado de educación secundaria: papel de las didácticas especiales'. Investigación en la escuela, No. 16, pp. 7-21.

GALTUNG, J. (1980). The true worlds. A transnational perspective. New York: The Free Press. 
GIL, D. (1988). El fracaso en la resolución de problemas de física: Una investigación orientada por nuevos supuestos. Enseñanza de las ciencias, 6(2), 131-146.

GIL, D. (1991). ¿Qué han de saber y saber hacer los profesores de ciencias? Enseñanza de las ciencias, 9(1) pp. 69-77.

GIORDAN, A. (1985). Enseñanza de las Ciencias, Madrid: Siglo XXI.

GLASERSFELD, E. von (1995). Radical constructivism: A Way of knowing and learning. London: The Falmer press.

GRUNDY, S. (1987). Curriculum: Productor praxis. London: The Falmer press.

GUBA, E. O., \& Lincoln, Y.S. (1989). Fourth generation evaluation, Newbury Park, CA: SAGE

HEWSON, P., \& Hewson, M. (1987). Science teachers conceptions of teaching: Implicatians tor teacher education. International Journal of Science Education, 9(4), pp. 425-440.

HODSON, D. (1985). Philosophy of Science, Science and Science Education. Studies in Science Education, 12, pp. 25-57.

McROBBIE, C., \& Tobin, K. (1995). Restraints to reform:The cangruence of teacher and student actions in a chemistry classroom. Journal of Research in Science Teaching, 32(4), 373-385.

PAYA, J. (1991). Los trabajos prácticos en la enseñanza de la Física y la Química. Un análisis critico y una propuesta fundamentada. Tesis doctoral. Universidad de Valencia, España.

POPE, M., \& Scott, E. (1983). La epistemología y la práctica de los profesores. Constructivismo y Enseñanza de las Ciencias, p. 179-191.

PORLAN, R. (1989). Teoría de Conocimiento. Teoría de la Enseñanza y Desarrollo Profesional: Las Concepciones Epistemológicas de los Profesores. Tesis de Doctorado. Universidad de Sevilla, España.

REYES, L (1993). Contructivist sugestions fo achieve science and technology education for all. Paper presented at the international forum for project 2000+helo at UNESCO headquarters, Paris, 5-10 July.

REYES, H. L. (1996) An interpretive study of social forces that constrain actions and interactions in a science classroom in Colombia. Doctoral dissertation. The Florida State University, Tallahassee, Florida.

RODRÍGUEZ, A. (1988). La educación Colombiana: datos y cifras. Educacion y Cultura, $16,5-14$.

SALCEDO, L (1991). Aprendizaje memorístico del concepto de equilibrio químico. VIII Congreso Colombiana de Química Cali Memorias, pp. 413-415. 
SALCEDO, L. y ERAZO, M. (1994). Concepciones de os profesores de química del nivel medio sobre prácticas de laboratorio. En Memorias Congreso Colombiano de Química Santa Maria.

SALCEDO. L, JESSUP, M. y PLAMEN, N. (1996) Evaluación en la enseñanza de las ciencias. Educación y cultura, No. 39, pp. 17-23

SCHIBECCI R, (1982). Attitudes to Science, update. Studies in Science Educafion, 11. pp. 26-59.

TOBIN, K. (1993) Constructivist perspectivas Qn teacher learning. In K. Tobin (Ed). The Practice of Constructivism in Science Education. Washington, D.C. AAA. Press. 